ISSN 2237-4876
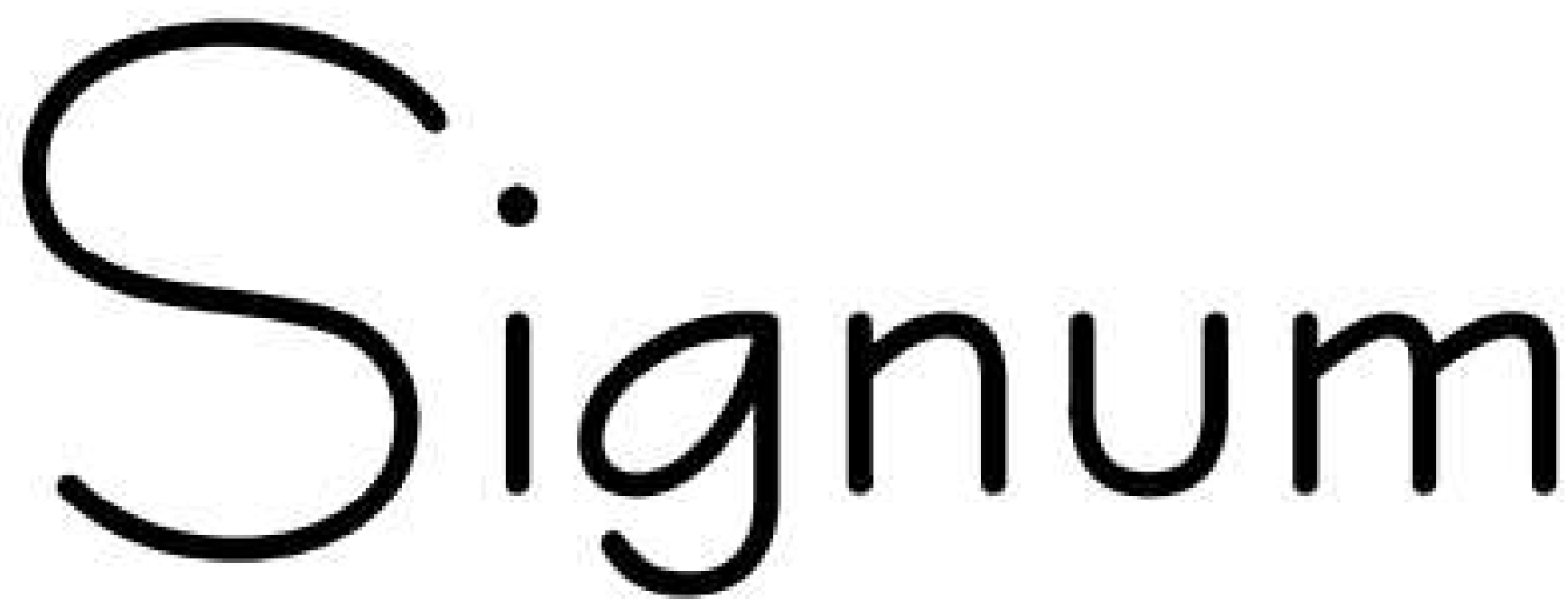

\title{
Estudos da Linguagem
}

Volume 23, Número 2 - Agosto de 2020

\section{Volume Temático}

Da Construção Textual à Análise do Discurso: DO PRIMEIRO GESTO À EXPLOSÃO DE SENTIDOS

\author{
Organização \\ Edina Regina Pugas Panichi \\ Esther Gomes de Oliveira \\ Maria Isabel Borges \\ Rosemeri Passos Baltazar \\ Livia Sprizão de Oliveira
}

Signum: Estudos da Linguagem, Londrina, v. 23, n. 2, p. 1-134, agosto de 2020 


\title{
Revista Signum: Estudos da Linguagem
}

Publicação Quadrimestral do

Programa de Pós-Graduação em Estudos da Linguagem

\section{Indexado por / Indexed by}

Sociological Abstracts

Linguistics and Language Behavior Abstracts - LLBA Abstracts

Red de Revistas Científicas de América Latina y el Caribe, España y Portugal - Redalyc

Directory of Open Access Journals - DOAJ

Diretório de Políticas de Acesso Aberto das Revistas Científicas Brasileiras - Diadorim IBICT

\author{
Redação \\ SIGNUM - Estudos da Linguagem \\ Programa de Pós-Graduação em Estudos da Linguagem \\ Universidade Estadual de Londrina \\ Centro de Letras e Ciências Humanas \\ Caixa Postal 6001 - CEP 86051-980 \\ Londrina, Paraná, Brasil \\ signum@uel.br
}

\section{Expediente}

Editoração Eletrônica: Leodmar Romam de Oliveira

Capa: Letícia Aparecida Nakayama

Foto da Capa: Célio Costa

Encurtador de URL: < bit.ly>

Signum: Estudos da linguagem / Centro de Letras e Ciências Humanas, Universidade Estadual de Londrina. - N. 1 (1998)-

Londrina: UEL, 1998-

Anual (até 2002) / Semestral (de 2003/1 a 2016/2)

Quadrimestral (a partir de 2017/1)

ISSN 1516-3083 (impressa até 2013)

ISSN 2237-4876 (on-line, a partir de 2011/1)

1. Linguagem - Periódicos. 2. Linguística - Periódicos. I. Universidade Estadual de Londrina. Centro de Letras e Ciências Humanas.

CDU 800(5) 
UNIVERSIDADE EsTAdUAL DE LONDRINA

Reitor: Sérgio Carlos de Carvalho

Vice-Reitor: Décio Sabbatini Barbosa

Centro de Letras e Ciências Humanas

Diretora: Viviane Aparecida Bagio Furtoso

Vice-Diretora: Ana Heloísa Molina

Programa de Pós-Graduação em Estudos da Linguagem

Coordenadora: Fabiane Cristina Altino

Vice-Coordenadora: Joyce Elaine de Almeida Baronas

EDITORA RESPONSÁVEL

Dircel Aparecida Kailer

Editoras Associadas

Fabiane Cristina Altino

Joyce Elaine de Almeida Baronas

Juliana Reichert Assunção Tonelli

Editora TÉCNICA

Livia Sprizão de Oliveira (LO)

Suporte TÉCnico

Ana Paula Silva 


\section{Conselho Editorial}

Afranio Gonçalves Barbosa (UFRJ, Brasil)

Aparecida Feola Sella (UNIOESTE, Brasil)

Aparecida Negri Isquerdo (UFMS, Brasil)

Catarina Vaz Rodrigues (UFES, Brasil)

Célia Brito (UFPA, Brasil)

Cláudia Regina Brescancini (PUC-RS, Brasil)

Clécio dos Santos Bunzen Junior (UNIFESP, Brasil)

Dermeval da Hora Oliveira (UFPB, Brasil)

Dioney Moreira Gomes (UnB, Brasil)

Domingos Sávio Siqueira (UFBA, Brasil)

Edna Maria Fernandes dos Santos Nascimento (UNIFRAN, Brasil)

Eliane Gouvêa Lousada (USP, Brasil)

Fernanda Coelho Liberali (PUC-SP, Brasil)

Florencia Miranda (Universidad Nacional de Rosario, Argentina)

Francisco Moreno Fernández (Harvard, EUA)

Gladis Massini-Cagliari (UNESP, Brasil)

Glaís Sales Cordeiro (Université de Genève, Suiça)

Gonzalo Navaza (Universidad de Vigo, Espanha)

Irene Maria Ferreira Blayer (Brock University, Canadá)

Izabel Christine Seara (UFSC, Brasil)

Juliano Desiderato Antonio (UEM , Brasil)

Lúcia Furtado de Mendonça Cyranka (UFJF, Brasil)

Luciana Fracasse (UNICENTRO, Brasil)

Mara Sofia de Toledo Zanotto (PUC-SP, Brasil)

Marcelo Módolo (USP, Brasil)

Maria Adelaide de Freitas (UEM, Brasil)

Maria Antónia Coutinho (Universidade Nova de Lisboa, Portugal)

Maria Cristina Faria Dalacorte Ferreira (UFG, Brasil)

Maria do Socorro Oliveira (UFRGS, Brasil)

Maria José Gnatta Dalcuche Foltran (UFPR, Brasil)

Maria Lúcia da Cunha Victório de Oliveira Andrade (USP, Brasil) 


$$
\begin{gathered}
\text { Maria Marta Furlanetto (UFSC, Brasil) } \\
\text { Marilda do Couto Cavalcanti (UNICAMP, Brasil) } \\
\text { Mariluci da Cunha Guberman (UFRJ, Brasil) } \\
\text { Marilucia Barros Oliveira (UFPA, Brasil) }
\end{gathered}
$$

Melba Libia Cárdenas Beltrán (Universidad Nacional de Colombia, Colômbia)

Nora Basurto Santos (Universidad Veracruzana, México)

Nukácia Meyre Silva Araújo (UECE, Brasil)

Odete Pereira da Silva Menon (UFPR, Brasil)

Paula Golombek (Florida State University, Estados Unidos)

$$
\text { Pedro Navarro (UEM, Brasil) }
$$

Regina Souza Gomes (UFRJ, Brasil)

Renilson José Menegassi (UEM, Brasil)

Roberto Leiser Baronas (UFSCar, Brasil)

Rosane de Andrade Berlinck (UNESP, Brasil)

Rosane de Sá Amado (USP, Brasil)

Rosario Álvarez (Universidade de Santiago de Compostela, Espanba)

Sanimar Busse (UNIOESTE, Brasil)

Sheila Elias de Oliveira (UNICAMP, Brasil)

Silvia Figueiredo Brandão (UFRJ, Brasil)

Sonia Maria Lazzarini Cyrino (UNICAMP, Brasil)

Stella Maris Bortoni de Figueiredo Ricardo (UnB, Brasil)

Terezinha da Conceição Costa-Hübes (UNIOESTE, Brasil)

Vanderci de Andrade Aguilera (UEL, Brasil)

Vandersí Sant'Ana Castro (UNICAMP, Brasil) 


\section{Pareceristas Ad Hoc}

Albano Dalla Pria (UNEMAT, Brasil)

André Coneglian (UFMG, Brasil)

Celia dos Santos (UEL, Brasil)

Daiany Bonácio (UEL, Brasil)

Eliana Maria Severino Donaio Ruiz (UEL, Brasil)

Eva Cristina Francisco (IFSP, Brasil)

Flávio Luis Freire Rodrigues (UEL, Brasil)

Isabel Cristina Cordeiro (UEL, Brasil)

Juliana dos Santos Barbosa (Pitágoras, Brasil)

Letícia Jovelina Storto (UENP, Brasil)

Lolyane Cristina Guerreiro de Oliveira (UEL, Brasil)

Marcelo Silveira (UEL, Brasil)

Miguel Luiz Contani (UEL, Brasil)

Sérgio Romaneli (UFSC, Brasil)

Sônia Aparecida Vido Pascolati (UEL, Brasil)

Paulo Roberto Almeida (UEL, Brasil)

Tânia Scoparo (UENP, Brasil) 